\title{
Long non-coding RNA MALAT1 sponges miR-124-3p.1/KLF5 to promote pulmonary vascular remodeling and cell cycle progression of pulmonary artery hypertension
}

\author{
DAPENG WANG ${ }^{1,2}$, HONGYANG XU ${ }^{2}$, BO WU $^{3}$, SHUYUN JIANG ${ }^{2}$, \\ HONG PAN $^{2}$, RUILAN WANG ${ }^{1}$ and JINGYU CHEN ${ }^{4}$
}

\author{
${ }^{1}$ Department of Critical Care Medicine, Shanghai General Hospital of Nanjing Medical University, \\ Shanghai 201620; Departments of ${ }^{2}$ Intensive Medicine and ${ }^{3}$ Lung Transplantation; ${ }^{4}$ Lung Transplant Group, \\ Wuxi People's Hospital Affiliated to Nanjing Medical University, Wuxi, Jiangsu 214021, P.R. China
}

Received February 15, 2019; Accepted June 19, 2019

DOI: $10.3892 / \mathrm{ijmm} .2019 .4256$

\begin{abstract}
Previous studies have demonstrated that long non-coding RNA (lncRNA) is involved in vascular remodeling. The metastasis-associated lung adenocarcinoma transcript 1 (MALAT1) lncRNA is associated with the proliferation and migration of vascular smooth muscle and endothelial cells; however, its biological role in pulmonary artery hypertension (PAH) is currently unclear. The aim of the present study was to investigate the post-transcriptional regulation of MALAT1 in human pulmonary artery smooth muscle cells (HPASMCs). The results revealed that MALAT1 expression levels were significantly upregulated in the pulmonary arteries (PAs) and HPASMCs obtained from patients with PAH compared with adjacent normal PA tissues and HPASMCs. Knockdown of MALAT1 suppressed the viability and proliferation of HPASMCs and prevented cells entering the $\mathrm{G}_{0} / \mathrm{G}_{1}$ cell cycle phase. MALAT1 overexpression exerted the opposite effects. Bioinformatics analysis predicted complementary binding of hsa-microRNA (miR)-124-3p.1 with the 3'-untranslated region of MALAT1. Luciferase reporter assays and RNA immunoprecipitation experiments demonstrated molecular binding between MALAT1 and hsa-miR-124-3p.1.
\end{abstract}

Correspondence to: Professor Ruilan Wang, Department of Critical Care Medicine, Shanghai General Hospital of Nanjing Medical University, 650 Xinsongjiang Road, Shanghai 201620, P.R. China

E-mail: wangyusun@hotmail.com

Professor Jingyu Chen, Lung Transplant Group, Wuxi People's Hospital Affiliated to Nanjing Medical University, 299 Qing Yang Road, Wuxi, Jiangsu 214021, P.R. China

E-mail: cjy_wx@163.com

Key words: pulmonary artery hypertension, metastasis-associated lung adenocarcinoma transcript 1 , human pulmonary artery smooth muscle cells, proliferation, hsa-microRNA-124-3p.1, Kruppel-like factor 5
This resulted in the formation of an RNA-induced silencing complex. In addition, Kruppel-like factor 5 (KLF5) was confirmed to be a target gene of MALAT1/hsa-miR-124-3p.1. MALAT1 silencing did not inhibit the proliferation and migration of HPASMCs following knockdown of hsa-miR-124-3p.1. In addition, MALAT1 knockdown was demonstrated to attenuate the expression of KLF5. Following MALAT1 knockdown, the expression level of KLF5 was rescued by inhibition of hsa-miR-124-3p.1 expression. The results of the current study indicate that the MALAT1/hsa-miR-124-3p.1/KLF5 axis may serve a key role in HPASMCs. In addition, the results contribute to what is known regarding the role of MALAT1 in PAH development and provide a novel theoretical basis for the development of new therapeutic interventions for patients with PAH.

\section{Introduction}

Pulmonary artery hypertension (PAH) is a life-threatening disease, characterized by progressive remodeling of the distal pulmonary arteries (PAs) due to increased cell proliferation and resistance to apoptosis. $\mathrm{PAH}$ can lead to right-sided heart failure and eventually death (1). At present, there is no cure for PAH, and the goal of treatment is limited to delaying or preventing its progression. Although significant advances have been made in understanding the cellular and molecular events underlying the pathogenesis of $\mathrm{PAH}$, it remains an incurable disease. Therefore, understanding the molecular mechanisms of vascular remodeling in PAH may facilitate the development of more effective therapeutic strategies that improve the prognosis and long-term survival of patients with PAH.

Long non-coding RNAs (lncRNAs) are a sequence of nucleotides $>200$ bp in length, which were once considered as transcriptional 'noise' with no protein-encoding functions (2). However, it is now evident that they are involved in a variety of biological processes, including cell differentiation, apoptosis and proliferation (3). Metastasis-associated lung adenocarcinoma transcript 1 (MALAT1) is a highly conserved lncRNA that has been implicated in different cancer types, such as non-small cell lung cancer (4), prostate cancer (5) and 
gastric cancer (6). Recently, MALAT1 has received attention from researchers due to its observed role in the progression of vascular endothelial cell dysfunction. One study demonstrated that MALAT1 dysregulation significantly increased the proliferation of vascular endothelial cells under hypoxic conditions (7). In addition, it was reported that MALAT1 regulates the proliferation of smooth muscle cells and inhibits cardiac hypertrophy in PAH mice following silencing of MALAT1 (8). Together, these results suggest that MALAT1 serves a latent biological role in the pathogenesis of hypoxia and PAH. Although our understanding of the role of MALAT1 in PAH is increasing, additional studies are required to further verify its role and underlying mechanisms in PAH.

In addition to lncRNA, microRNAs (miRNAs/miRs) are a class of non-coding single-stranded RNA molecules that are $\sim 22$ nucleotides in length. They function as key regulators of gene expression in various biological processes at the transcriptional or post-transcriptional level through targeting mRNAs for degradation or suppressing translation (9). LncRNAs interact with miRNAs and regulate each other's levels of expression and biological activities. In addition, IncRNAs may function as competing endogenous RNAs (ceRNAs) that inhibit miRNA expression and activity (10). Of particular note, it has been reported that MALAT1 harbors binding sites for several miRNAs, such as miR-125b (11), miR-101b (12) and miR-183 (13). However, there is limited research regarding the interactions between MALAT1 and miRNAs in PAH.

The aim of the present study was to identify the molecular mechanisms of MALAT1 in the pathogenesis of PAH. The results demonstrated that the expression of MALAT1 was upregulated in PA tissues and human pulmonary artery smooth muscle cells (HPASMCs) of patients with PAH. Silencing of MALAT1 inhibited HPASMC proliferation. The effect of MALAT1 overexpression exerted the opposite effects. In addition, MALAT1 was demonstrated to function as a ceRNA for hsa-miR-124-3p.1. MALAT1 participates in pulmonary vascular remodeling by binding to hsa-miR-124-3p.1 to form a molecular sponge. This promotes the expression of the downstream target gene, Kruppel-like factor 5 (KLF5), which is also involved in pulmonary vascular remodeling progression (14). The results of the current study contribute to what is currently known regarding the role of MALAT1 in PAH and may provide new therapeutic targets and biomarkers for the treatment of this disease.

\section{Materials and methods}

Patient criteria. Patients diagnosed with PAH that had undergone lung transplantation surgery at Wuxi People's Hospital Affiliated to Nanjing Medical University (Wuxi, China) between October 2014 and October 2017 were included in the present study (Table I). Patients were 18-55 years of age (mean \pm standard deviation, $40 \pm 4$ years) with a 6 -min walking distance of $\geq 100$ and $<500 \mathrm{~m}$. PAH diagnosis was confirmed by echocardiography and the diagnostic procedures conformed to the 2015 European Society of Cardiology and European Respiratory Society guidelines for the diagnosis of pulmonary arterial hypertension, pulmonary hypertension due to left heart disease, pulmonary hypertension due to lung diseases and/or hypoxia, chronic thromboembolic pulmonary hypertension, other PA obstructions and pulmonary hypertension with unclear and/or multifactorial mechanisms. Patients with severe obstructive pulmonary disease, psychosis, a history of drug addiction or other diseases (chronic liver disease, portal hypertension, chronic kidney disease, amyloidosis and so on) and that had received prostacyclin, endothelin receptor antagonist, L-arginine or sildenafil were excluded.

Preparation of human lung tissue samples. Human PAH lung tissue samples were obtained from lung transplantation patients at the Lung Transplant Group in Wuxi People's Hospital Affiliated to Nanjing Medical University. This study was approved by the Ethics Committee for Use of Human Samples of the Nanjing Medical University, which was in accordance with The Code of Ethics of the Helsinki Declaration of World Medical Association for experiments involving humans. Each individual gave written informed consent prior to their participation. Healthy lung tissue samples were obtained from donors that were not suitable for transplantation. The inclusion criteria for donors were as follows: $<50$ years of age; a smoking history of $<20$ packs of cigarettes per year; no chest trauma or sustained mechanical ventilation for $<1$ week; $\mathrm{FiO}_{2}, 1.0$; positive end-expiratory pressure, $5 \mathrm{~cm} ; \mathrm{PaO}_{2},>300 \mathrm{mmHg}$; chest films indicating relatively clear lung fields; and bronchoscopy results indicating a clear trachea. Lung tissue collection and distal PA microscopic separation was performed according to the protocol described previously $(15,16)$.

Hematoxylin and eosin $(H \& E)$ staining. Human lung tissue samples were sectioned into tissue blocks and fixed in $4 \%$ paraformaldehyde overnight at $4{ }^{\circ} \mathrm{C}$. Tissues were then dehydrated, cleared and embedded in paraffin. The paraffin blocks were cut into $4-\mu \mathrm{m}$-thick sections and used for $\mathrm{H} \& \mathrm{E}$ staining analysis (17). The sections were stained with hematoxylin for $10 \mathrm{~min}$ at room temperature. The slices were rinsed with water for $2 \mathrm{~min}$ and transferred to differentiation fluid (1\% hydrochloric acid) for $30 \mathrm{sec}$. Subsequently, the slices were place in running water and washed for 30-60 min. Finally, the slices were stained with eosin for 2-5 min at room temperature and then washed. Images were captured using a light microscope (magnification, $\mathrm{x} 10$ ).

Culture of HPASMCs. Primary HPASMCs were isolated from the explanted lungs of patients undergoing transplantation for PAH, and from the PAs of donor lungs. The primary culture of HPASMCs was performed according to a previously published protocol (17). Using a light microscope, the distal PAs were microdissected from lung explant tissues in a dissection dish containing cold PBS. The separation steps were performed according to a previously published study (18). The HPASMCs were subsequently transferred to culture plates and incubated in SmGM-2 Smooth Muscle Growth medium-2 BulletKit media (Lonza Group, Ltd.) containing 10\% (volume/volume) heat-inactivated FBS (Gibco; Thermo Fisher Scientific, Inc.), $2 \mathrm{ng} / \mathrm{ml}$ human recombinant fibroblast growth factor, $0.5 \mathrm{ng} / \mathrm{ml}$ human recombinant epidermal growth factor, $50 \mu \mathrm{g} / \mathrm{ml}$ gentamicin and $5 \mu \mathrm{g} / \mathrm{ml}$ insulin. Cells were maintained at $37^{\circ} \mathrm{C}$ and $5 \% \mathrm{CO}_{2}$ in an incubator for 1 week (19). Passage 4-8 cells were used for subsequent experiments. 
Table I. Characteristics of PAH patients and healthy donor.

\begin{tabular}{|c|c|c|c|c|c|}
\hline Patient ID & Age, years & Sex & Ethnicity & Diagnosis/cause of death & $\mathrm{PAP}, \mathrm{mm} / \mathrm{Hg}$ \\
\hline PAH-01 & 55 & Female & Asian & НРAH & 90 \\
\hline PAH-02 & 43 & Male & Asian & HРAH & 115 \\
\hline PAH-03 & 50 & Male & Asian & НРAH & 94 \\
\hline PAH-04 & 48 & Male & Asian & HРAH & 89 \\
\hline PAH-05 & 47 & Female & Asian & НРАН & 112 \\
\hline PAH-06 & 48 & Female & Asian & HРAH & 91 \\
\hline PAH-07 & 52 & Female & Asian & НРAH & 90 \\
\hline PAH-08 & 51 & Male & Asian & НPAH & 108 \\
\hline Healthy donor-01 & 50 & Female & Asian & Donor lung tissue & $\mathrm{N} / \mathrm{A}$ \\
\hline Healthy donor-02 & 43 & Female & Asian & Donor lung tissue & N/A \\
\hline Healthy donor-03 & 48 & Female & Asian & Donor lung tissue & N/A \\
\hline Healthy donor-04 & 47 & Male & Asian & Donor lung tissue & N/A \\
\hline Healthy donor-05 & 44 & Male & Asian & Donor lung tissue & $\mathrm{N} / \mathrm{A}$ \\
\hline Healthy donor-06 & 52 & Male & Asian & Donor lung tissue & N/A \\
\hline Healthy donor-07 & 55 & Female & Asian & Donor lung tissue & $\mathrm{N} / \mathrm{A}$ \\
\hline Healthy donor-08 & 53 & Male & Asian & Donor lung tissue & N/A \\
\hline
\end{tabular}

HPAH, hereditary pulmonary artery hypertension; N/A, data not available; PAP, pulmonary artery pressure.

Immunofluorescence analysis. To examine the purity of HPASMCs, the expression of smooth muscle myosin heavy chain (SM-MHC) was analyzed by immunofluorescence staining. The cells were fixed with $4 \%$ polyformaldehyde for $15 \mathrm{~min}$ at $37^{\circ} \mathrm{C}$, washed three times with cold PBS for $5 \mathrm{~min}$ each time and then permeabilized with $0.5 \%$ Triton X-100 (Sigma-Aldrich; Merck KGaA) for $10 \mathrm{~min}$. The cells were blocked in 5\% bovine serum albumin (BSA) for $1 \mathrm{~h}$ at room temperature and incubated overnight at $4^{\circ} \mathrm{C}$ with SM-MHC antibody (cat. no. 21404-1-AP; 1:200; ProteinTech Group, Inc.), and subsequently stained with a Fluorescein-conjugated Affinipure Goat Anti-Rabbit IgG (H+L) secondary antibody (cat. no. SA00003-2; 1:100; ProteinTech Group, Inc.) for $1 \mathrm{~h}$ at room temperature. Slides were then incubated for $5 \mathrm{~min}$ with DAPI. Finally, cell staining was visualized by fluorescence microscopy.

Transfection. A short-hairpin RNA (shRNA) sequence targeting MALAT1 and a negative control sequence were obtained from Shanghai GenePharma Co., Ltd. The hsa-miR124-3p.1 mimic, mimic negative control, inhibitor and inhibitor negative control were also purchased from Shanghai GenePharma Co., Ltd. The sequences for each were as follows: hsa-miR124-3p.1 mimic, sense, 5'-UAAGGCACGCGGUGA AUGCCA-3'; miR-124 antisense, 3'-UAAUUCCGUGCGCCA CUUACG-5'; hsa-miR124-3p.1 inhibitor, 5'-GGCAUUCAC CGCGUGCCUUA-3'; sh-MALAT1, 5'-GAGTTGTGCTGC TATCTTA-3'. hsa-miR124-3p.1 mimic and inhibitor were transfected at a concentration of $50 \mu \mathrm{M}$. The mimic negative control and inhibitor negative control were transfected at a concentration of $50 \mu \mathrm{M}$. The overexpression plasmid containing the MALAT1 sequence (pcDNA-MALAT1) and empty vector (pcDNA) were synthesized by Shanghai GenePharma Co., Ltd. HPASMCs were transfected with
shMALAT1 and the shRNA negative control at a final concentration of $20 \mathrm{nmol} / \mathrm{l}$ using Lipofectamine ${ }^{\circledR} 2000$ (Invitrogen; Thermo Fisher Scientific, Inc.), according to the manufacturer's protocol. Furthermore, HPASMCs were transfected with pcDNA-MALAT1 and pcDNA at a final concentration of $5 \mathrm{mg} / \mathrm{l}$ using Lipofectamine $2000^{\circledR}$, according to the manufacturer's protocol. After 6-8 h, the transfection reagents were removed and the cells were further cultured in DMEM containing 5\% FBS for $24 \mathrm{~h}$ prior to subsequent experiments.

Reverse transcription-quantitative PCR (RT-qPCR). Total RNA was extracted from PAs and HPASMCs using TRIzol reagent (Thermo Fisher Scientific, Inc.). First strand cDNA synthesis was achieved using a high capacity cDNA reverse transcription kit (Applied Biosystems; Thermo Fisher Scientific, Inc.). Reactions were performed for $30 \mathrm{~min}$ at $60^{\circ} \mathrm{C}$, followed by heat inactivation for $2 \mathrm{~min}$ at $94^{\circ} \mathrm{C}$. miRNA was extracted using a miRcute Plus miRNA First-Strand cDNA Synthesis kit (Tiangen Biotech, Co., Ltd.). qPCR analysis was performed using FastFire qPCR PreMix (SYBR-Green; Tiangen Biotech, Co., Ltd.) and the ABI StepOne Real-Time PCR System (Applied Biosystems; Thermo Fisher Scientific, Inc). Reactions were performed using the following thermocycling conditions: Pre-denaturation at $95^{\circ} \mathrm{C}$ for $1 \mathrm{~min}$; 40 cycles of $95^{\circ} \mathrm{C}$ for $5 \mathrm{sec}, 60^{\circ} \mathrm{C}$ for $15 \mathrm{sec}$, and a final step at $72^{\circ} \mathrm{C}$ for $15 \mathrm{sec}$. GAPDH, $\beta$-actin and U6 were used as endogenous controls for MALAT1, KLF5 and hsa-miR-124-3p.1 expression analysis. The primers used were as follows: MALAT1 forward, 5'-GCAGGCGTTGTGCGTAGAG-3', and reverse, 5'-TTGCCGACCTCACGGATT-3'; GAPDH forward, 5'-CGC ATTGCCAGACATATCAGC-3', and reverse, 5'-AGGTGA AGCAGGCTCAATCAA-3'; hsa-miR-124-3p.1 forward, 5'-ACACTCCAGCTGGGTAAGGCACGCGGTG-3', and reverse, 5'-TGGTGTCGTGGAGTCG-3'; U6 forward, 5'-CTC 
GCTTCGGCAGCACA-3', and reverse, 5'-TGGTGTCGTGGA GTCG-3'; KLF5 (212 bp) forward, 5'-AGCTCACCTGAG GACTCATA-3', and reverse, 5'-GTGCGCAGTGCTCAGT TCT-3'; and $\beta$-actin, forward, 5'-TGAGAGGGAAATCGT GCGTGAC-3', and reverse, 5'-AAGAAGGAAGGCTGGAAA AGAG-3'. Relative target expression levels were calculated using the $2^{-\Delta \Delta \mathrm{Ct}}$ method (20).

Western blotting. Protein samples were extracted from PAs and HPASMCs by lysing them in RIPA buffer for $30 \mathrm{~min}$ on ice. Samples were then sonicated with $20 \mathrm{KHz}$ frequency on ice for $1 \mathrm{~min}$ and centrifuged at $14,000 \mathrm{xg}$ for $15 \mathrm{~min}$ at $4^{\circ} \mathrm{C}$. The protein concentration was quantified using a BCA kit (Nanjing KeyGen Biotech Co., Ltd.). Total protein $(20 \mu \mathrm{g})$ was separated by $10 \%$ SDS-PAGE, transferred to nitrocellulose membranes and subjected to western blotting according to the previously published protocols $(15,16)$. Nitrocellulose membranes were incubated at $4^{\circ} \mathrm{C}$ overnight with the following specific antibodies: proliferating cell nuclear antigen (PCNA; cat. no. 10205-2-AP; 1:1,000; ProteinTech Group, Inc.), cyclin A1 (cat. no. 13295-1-AP; 1:1,000; ProteinTech Group, Inc.), KLF5 (cat. no. ab137676; 1:500; Abcam), cyclin D1 (cat.no. 60186-1-lg; 1:1,000; ProteinTech Group, Inc.), cyclin E1 (cat. no. 11554-1-AP; 1:1,000; ProteinTech Group, Inc.) and $\beta$-actin (cat. no. 60008-1-lg; 1:5,000; ProteinTech Group, Inc.). Membranes were subsequently incubated with goat-anti-rabbit IgG secondary antibodies (cat. no. SA00001-2; 1:10,000; Nanjing KeyGen Biotech Co., Ltd.) or goat-anti-mouse IgG secondary antibody (cat. no. SA00001-1; 1:10,000; Nanjing KeyGen Biotech Co., Ltd), shaken and incubated at room temperature for $1 \mathrm{~h}$. Imaging was then performed using the ECL plus detection reagent (Thermo Fisher Scientific, Inc.) Blots were imaged by VersaDoc ${ }^{\mathrm{TM}}$ MP 4000 (Bio-Rad Laboratories, Inc.) and quantified using PDQuest Advanced 2D analysis software (version 8.0; Bio-Rad Laboratories, Inc.).

Cell Counting kit-8 (CCK-8) assay. To examine the rate of cell proliferation, HPASMCs were seeded at a density of $0.5 \times 10^{4}$ cells in 96-well microtiter plates and cultured without serum for $24 \mathrm{~h}$. The cells were then transfected with the aforementioned sequences. Following incubation at $37^{\circ} \mathrm{C}$ for $48 \mathrm{~h}, 20 \mu \mathrm{l} \mathrm{CCK}-8$ reagent was added and the cells were incubated for a further $4 \mathrm{~h}$ at $37^{\circ} \mathrm{C}$. The absorbance was read at $450 \mathrm{~nm}$ using a Synergy ${ }^{\mathrm{TM}}$ 2 multifunctional enzyme standard instrument.

Cell cycle analysis. To investigate the role of MALAT1 in cell cycle progression, the cell cycle distribution of HPASMCs transfected with sh-MALAT1 or pcDNA-MALAT1 was analyzed by flow cytometry. Following transfection, cells were collected by trypsinization, centrifuged at $425 \mathrm{x}$ g for $5 \mathrm{~min}$ at room temperature, and then resuspended in $1 \mathrm{ml}$ cold PBS. The cells were then washed twice with PBS and fixed in $70 \%$ ethanol at $4^{\circ} \mathrm{C}$ overnight. Cell fragments were resuspended in $200 \mu \mathrm{l}$ PBS and $200 \mu 1$ RNase A for $10 \mathrm{~min}$ at room temperature. At this point, the cells were stained with $200 \mu \mathrm{l}$ propidium iodide at $4^{\circ} \mathrm{C}$ for $10 \mathrm{~min}$ in the dark. HPASMCs were then filtered once through 400-mesh sieves before they were analyzed using a FACSCalibur Flow Cytometer (BD Biosciences). The data were analyzed using ModFit software (version 4.1; Verity Software House, Inc., Topsham, ME, USA).
Target prediction and luciferase reporter gene activity assay. TargetScan 7.2 (http://www.targetscan.org/) and DIANA-LncBase (http://omictools.com/diana-lncbase-tool) were used for target prediction. The wild-type or mutant 3'-untranslated region (3'-UTR) of MALAT1 containing the predicted hsa-miR-124-3p.1 binding site was purchased from Shanghai GenePharma Co., Ltd. and inserted into the pmirGLO Dual-Luciferase miRNA Target Expression Vector (Promega Corporation). Similarly, the 3'-UTR of KLF5 containing the predicted target sites for hsa-miR-124-3p.1 was synthesized by PCR amplification. This fragment was inserted into the pmirGLO dual luciferase miRNA target expression vector (Promega Corporation) to generate the KLF5-wild type reporter vector. This vector and hsa-miR-124-3p.1 were subsequently co-transfected into HPASMCs using Lipofectamine $2000^{\circledR}$ transfection reagent (Thermo Fisher Scientific, Inc.). After 6-8 h, the transfection reagents were removed and the HPASMCs were further cultured in DMEM containing 5\% FBS for $48 \mathrm{~h}$. Luciferase activity was measured using a dual luciferase reporter assay (Promega Corporation). Renilla luciferase activity was used for normalization.

RNA immunoprecipitation (RIP) assay. An RIP assay was used to investigate the interaction between MALAT1 and hsa-miR-124-3p.1 using the EZ-Magna RIP ${ }^{\mathrm{TM}}$ RNA Binding Protein Immunoprecipitation kit (Merck KGaA). The cells were first lysed using the lysis buffer (catalog no. 17-701; Merck KGaA) before they were incubated with an anti-human argonaute RNA-induced silencing complex (RISC) catalytic component 2 (AGO2) antibody (Merck KGaA) coated on magnetic beads in RIP buffer. Input and normal $\mathrm{IgG}$ were selected as controls for the experiment. RNA was isolated and reverse transcribed into cDNA before MALAT1 and hsa-miR-124-3p.1 levels were analyzed by RT-qPCR.

Scratch wound healing assay. To determine cell migration, HPASMCs were transferred into 6-well plates and cultured to $90 \%$ confluence. A sterile pipette tip was then used to generate a vertical 'wound' $\sim 1 \mathrm{~mm}$ in diameter. Cell migration in the 6-well plates was measured as described previously (15). HPASMCs were washed with cold PBS and images were captured under a light microscope to record the wound width at $0 \mathrm{~h}$. The culture medium was then replaced with medium containing 5\% FBS. Following incubation for $24 \mathrm{~h}$, the cell images were again captured under a microscope and the degree of migration was marked to quantify the cell migration ability.

Statistical analysis. Experiments were repeated at least three times and all data were presented as the mean \pm standard error of mean. Statistical differences between or among groups were analyzed using a Student's t-test or one-way ANOVA followed by Bonferroni's test using the GraphPad Prism software package (version 5.0; GraphPad Software, Inc.). $\mathrm{P}<0.05$ was considered to indicate a statistically significant difference.

\section{Results}

MALAT1 is highly expressed in PA tissues and HPASMCs derived from patients with $P A H$. The vascular wall morphology of the lung tissue samples was analyzed by H\&E staining. 
A

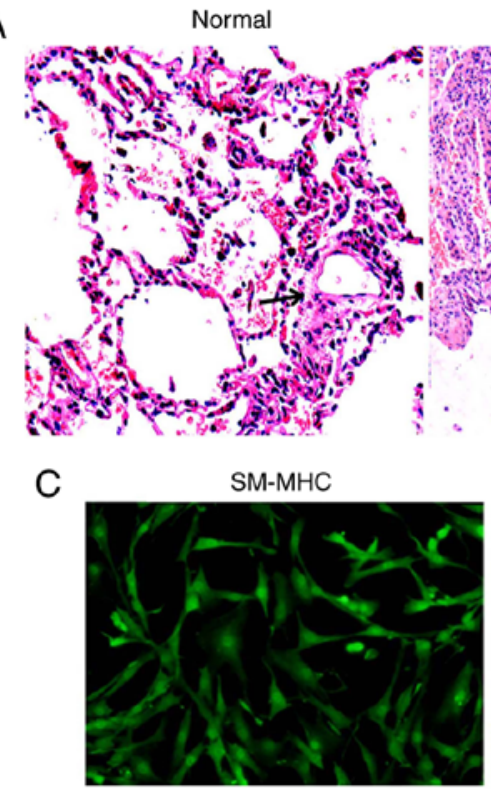

D

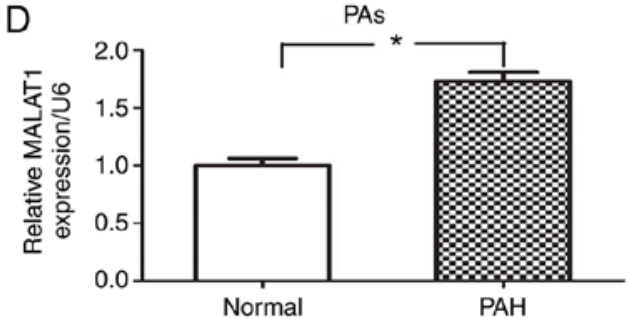

$\mathrm{PAH}$

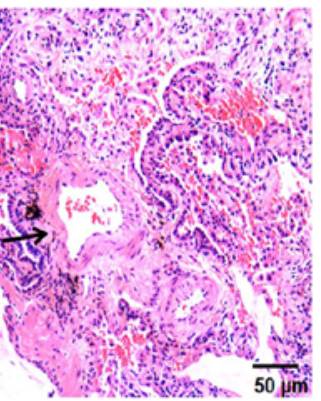

DAPI
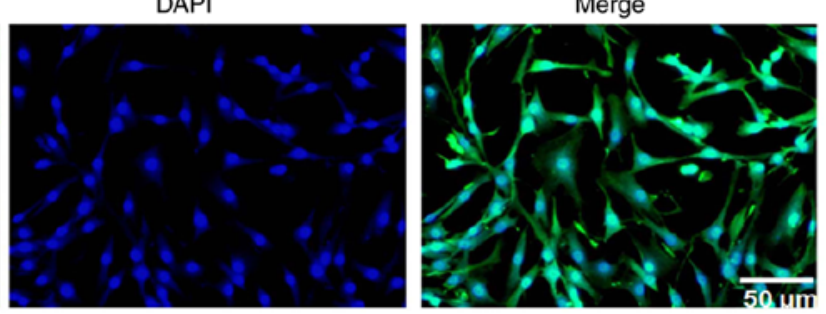

$\mathrm{E}$

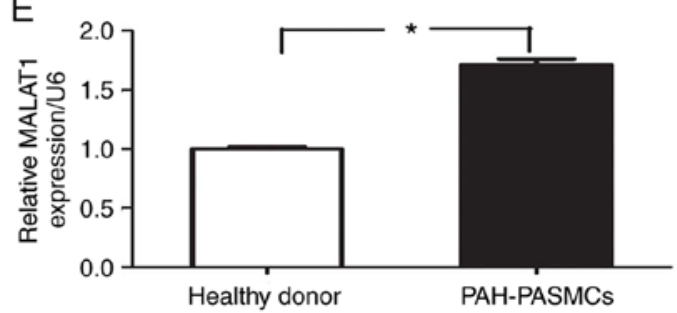

Figure 1. MALAT1 is upregulated in PA tissues and HPASMCs from patients with PAH. (A) Hematoxylin \& eosin staining of human PAs indicated that the wall thickness in PAH samples was increased compared with the normal controls. Arrows indicate the morphology of PAs in patients with PAH and healthy lung donors $(\mathrm{n}=8$; scale bar, $50 \mu \mathrm{m})$. (B) Histogram showing that the wall thickness was increased in patients with PAH compared with normal controls. (C) Immunofluorescence analysis of the expression of SM-MHC verifying the purity of HPASMCs. The expression level of MALAT1 in (D) PA tissues and (E) HPASMCs derived from healthy donors and patients with PAH, as determined by reverse transcription-quantitative PCR analysis. ${ }^{*}<0.05$. MALAT1, metastasis-associated lung adenocarcinoma transcript 1; PA, pulmonary artery; HPASMCs, human pulmonary artery smooth muscle cells; PAH, pulmonary arterial hypertension; SM-MHC, smooth muscle myosin heavy chain.

Compared with the healthy volunteers, the vascular wall thickness of patients with PAH was significantly increased in medium-sized PAs $(\mathrm{P}<0.05$; Fig. $1 \mathrm{~A}$ and $\mathrm{B})$. The purity of HPASMCs was verified using smooth muscle myosin heavy chain antibody (Fig. 1C). To determine whether MALAT1 may be involved in the pathologic process of PAH, a total of eight paired PAH and normal PA tissue samples from patients with PAH were used to determine the expression of MALAT1 by RT-qPCR analysis. As demonstrated in Fig. 1D, the average expression level of MALAT1 in PAs from PAH tissues was significantly increased compared with the normal PAs tissues $(\mathrm{P}<0.05)$. In addition, RT-qPCR analysis revealed that the expression of MALAT1 was significantly upregulated in HPASMCs from patients with PAH compared with those from healthy donors $(\mathrm{P}<0.05$; Fig. 1E). These results suggest that MALAT1 is highly expressed in PA tissues and HPASMCs derived from patients with PAH.

MALAT1 affects the proliferation and migration of HPASMCs. To verify the role of MALAT1 in the cellular processes of HPASMCs, the proliferation and migration of HPASMCs transfected with sh-MALAT1 and pcDNA-MALAT1 were analyzed. As demonstrated in Figs. 2 and 3, the transfection efficiency of sh-MALAT1 and pcDNA-MALAT1 was confirmed by
RT-qPCR analysis. A CCK-8 assay was then used to determine the growth rate of HPASMCs. The results demonstrated that knockdown of MALAT1 significantly inhibited the growth of HPASMCs when compared with the negative control group $(\mathrm{P}<0.001$; Fig. 2B). By contrast, MALAT1 overexpression promoted cell proliferation when compared with the negative control group (Fig. 3B). To examine whether MALAT1 may regulate cell cycle progression, flow cytometry was used to analyze the cell cycle distribution. The results indicated that MALAT1 silencing reduced the percentage of cells (Fig. 2C and D), while MALAT1 overexpression increased the percentage of cells (Fig. 3C and D), in the $\mathrm{G}_{2} / \mathrm{M}$ and $\mathrm{S}$ phases. It has been reported that cell cycle proteins (such as: Cyclin A1/D1/E1) serve key roles in $\mathrm{S}$ and $\mathrm{G}_{2} / \mathrm{M}$ phases, and have been widely recognized as markers of cell proliferation $(21,22)$. To understand the effect of MALAT1 on the regulation of cyclin proteins, western blotting was performed to analyze the expression levels of PCNA, cyclin A1, cyclin D1 and cyclin E1. The results demonstrated that transfection of HPASMCs with sh-MALAT1 significantly inhibited the expression of cell cycle proteins, PCNA, cyclin A1, cyclin D1 and cyclin E1 when compared with the controls $(\mathrm{P}<0.01$; Fig. 2E). By contrast, MALAT1 overexpression significantly increased the expression levels of these proteins when compared with the controls $(\mathrm{P}<0.01$; Fig. $3 \mathrm{E})$. 

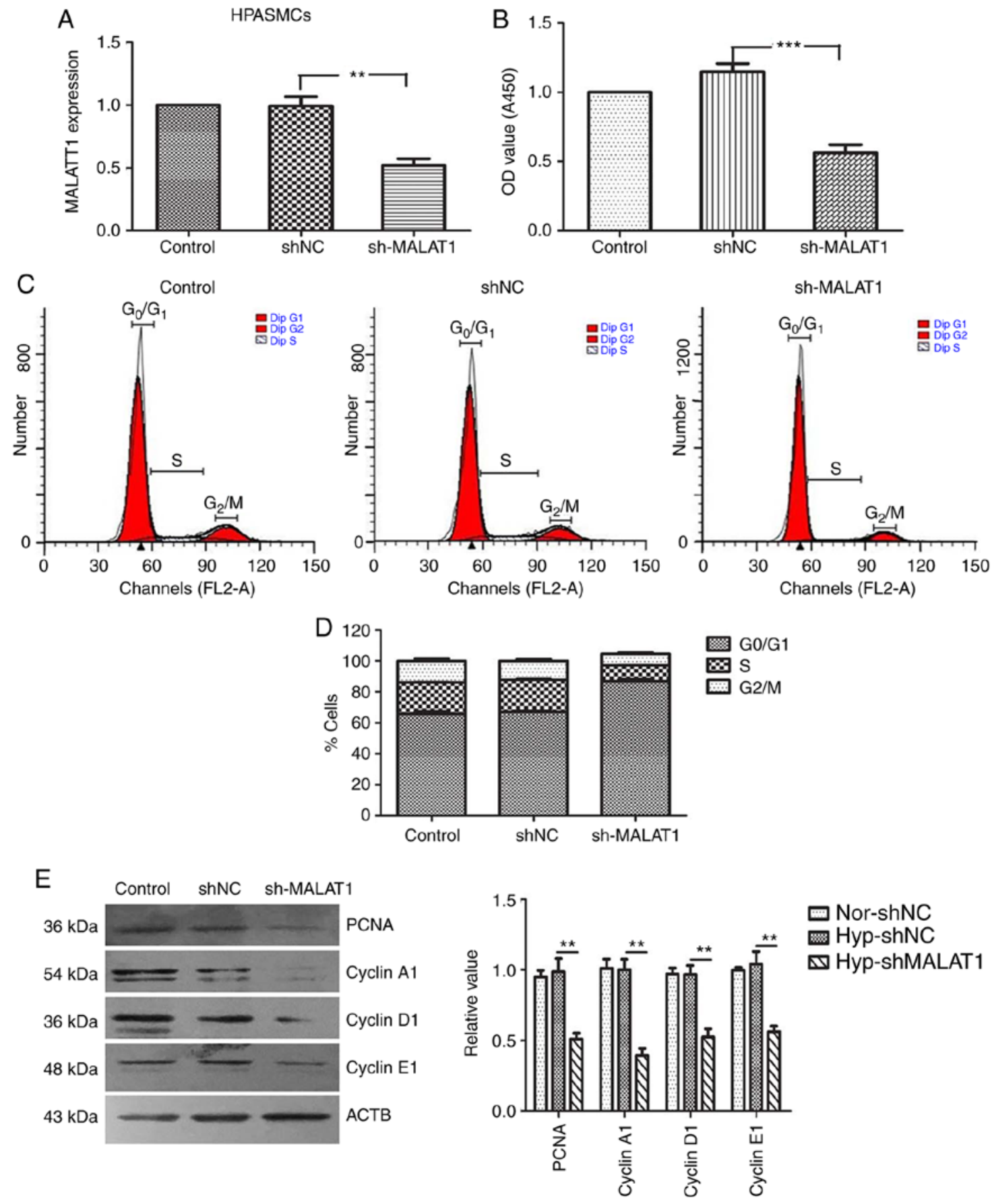

Figure 2. MALAT1 silencing reduces the proliferation of HPASMCs. (A) Transfection efficiency was examined by reverse transcription-quantitative PCR. Changes in (B) cell viability and (C) cell cycle distribution were assessed and (D) analyzed quantitatively. (E) Protein expression levels of PCNA, cyclin A1, cyclin D1 and cyclin E1 were analyzed by western blotting following transfection with sh-MALAT1. ${ }^{* *} \mathrm{P}<0.01$ and ${ }^{* * *} \mathrm{P}<0.001$. MALAT1, metastasis-associated lung adenocarcinoma transcript 1; HPASMCs, human pulmonary artery smooth muscle cells; PCNA, proliferating cell nuclear antigen; sh, short-hairpin RNA; OD, optical density.

To examine whether MALAT1 may affect the migratory ability of HPASMCs in vitro, the role of MALAT1 in HPASMC cell migration was analyzed using a scratch wound healing assay. The results demonstrated that MALAT1 silencing significantly inhibited cell migration in vitro when compared with the negative control group ( $\mathrm{P}<0.001$; Fig. $4 \mathrm{~A}$ and $\mathrm{B})$. By contrast, MALAT1 overexpression significantly promoted the migration of HPASMCs ( $\mathrm{P}<0.001$; Fig. $4 \mathrm{C}$ and $\mathrm{D})$. These results suggest that increased expression of MALAT1 may be a lethal risk factor for PAH and that a reduction in MALAT1 expression may exert a protective effect on the occurrence and development of the disease.

MALAT1 functions as a ceRNA that binds directly to hsa-miR-124-3p. LncRNAs may function as ceRNAs in different types of carcinomas (23). Previous studies have also indicated that MALAT1 may promote bladder cancer (11), liver fibrosis (12) and melanoma (13) progression by functioning as a ceRNA that targets miR-125b, miR-101b and miR-183, respectively. PAH and cancer share the common 

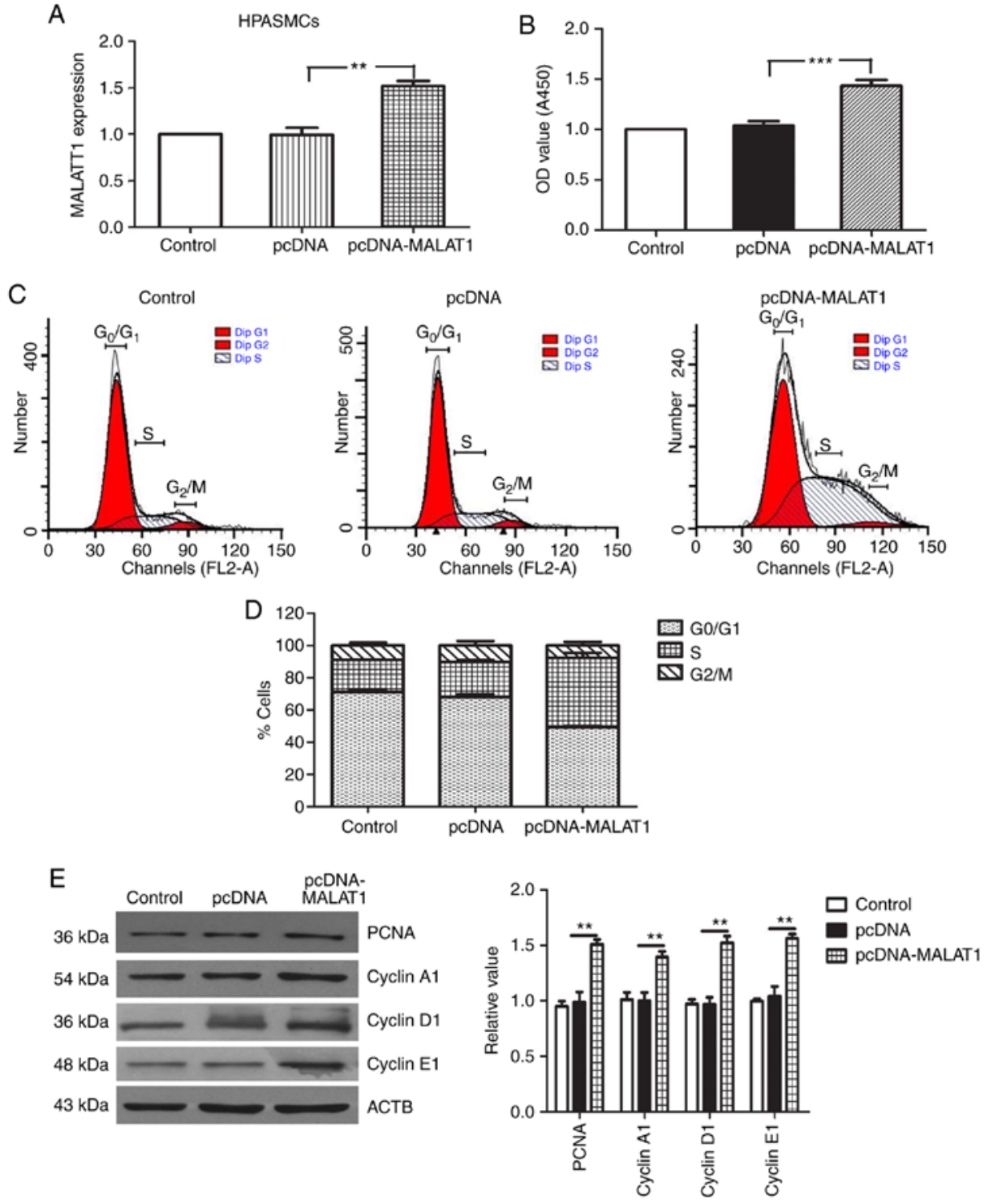

Figure 3. MALAT1 overexpression promotes the proliferation of HPASMCs. (A) Transfection efficiency was examined by reverse transcription-quantitative PCR. Changes in (B) cell viability and (C) cell cycle distribution were assessed and (D) analyzed. (E) Protein expression levels of PCNA, cyclin A1, cyclin D1 and cyclin E1 were analyzed by western blotting following transfection with pcDNA-MALAT1. ${ }^{* *} \mathrm{P}<0.01$ and ${ }^{* * *} \mathrm{P}<0.001$. MALAT1, metastasis-associated lung adenocarcinoma transcript 1; HPASMCs, human pulmonary artery smooth muscle cells; PCNA, proliferating cell nuclear antigen; OD, optical density.

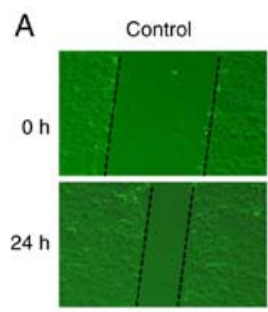

C

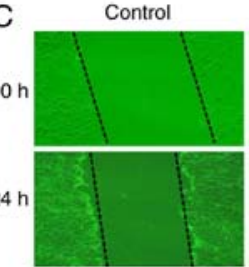

shNC

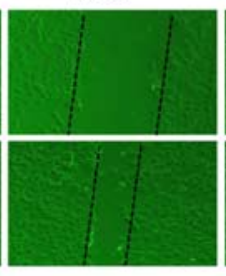

PcDNA

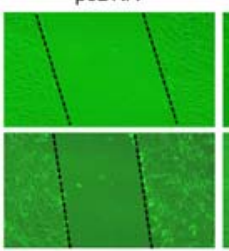

Sh-MALAT1

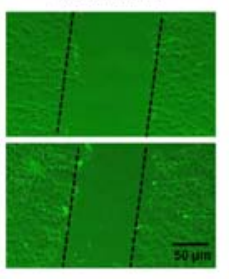

PCDNA-MALAT1

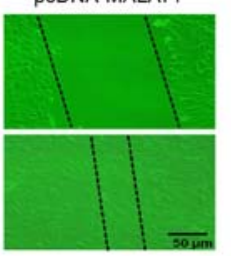

B
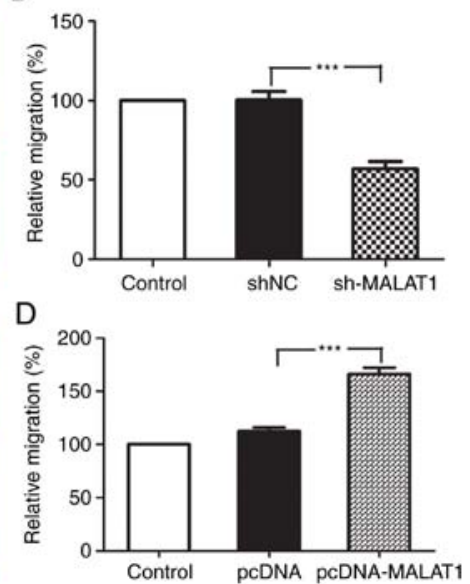

Figure 4. MALAT1 affects the migration of HPASMCs. HPASMCs were analyzed using cell migration assays following transfection with (A) shNC or sh-MALAT1 and (B) quantification of the results. HPASMCs were analyzed using cell migration assays following transfection with (C) pcDNA or pcDNA-MALAT1 and (D) quantification of the results $(n=6) .{ }^{* * *} \mathrm{P}<0.001$. MALAT1, metastasis-associated lung adenocarcinoma transcript 1 ; HPASMCs, human pulmonary artery smooth muscle cells; sh, short-hairpin RNA; NC, negative control. 
A

hsa-miR-124-3p. 1 3'...CCGUAAGUGGCGCACGGAAU...5' |II|||

MALAT1 $4247 \ldots . .$. CUAAAGUGAUCAGUGCCUUG...4267

hsa-miR-124-3p.1 3'...CCGUAAGUGGCGCACGGAAU...5

||||||

MALAT1 6368...CAUCGCCACCCCGUGCCUUU...6388
$\mathrm{B}$

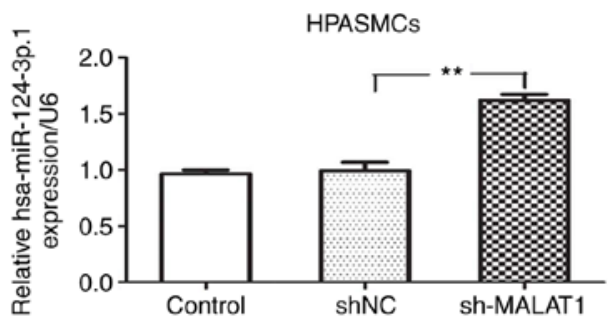

D - HPASMCS

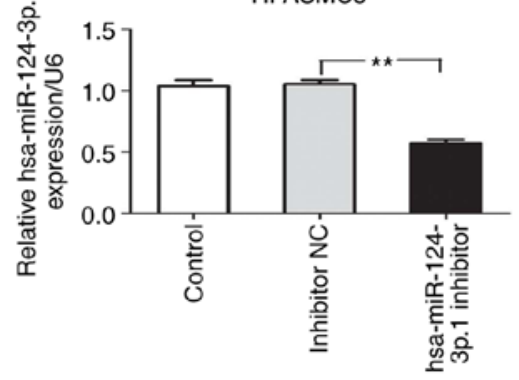

$\mathrm{E}$
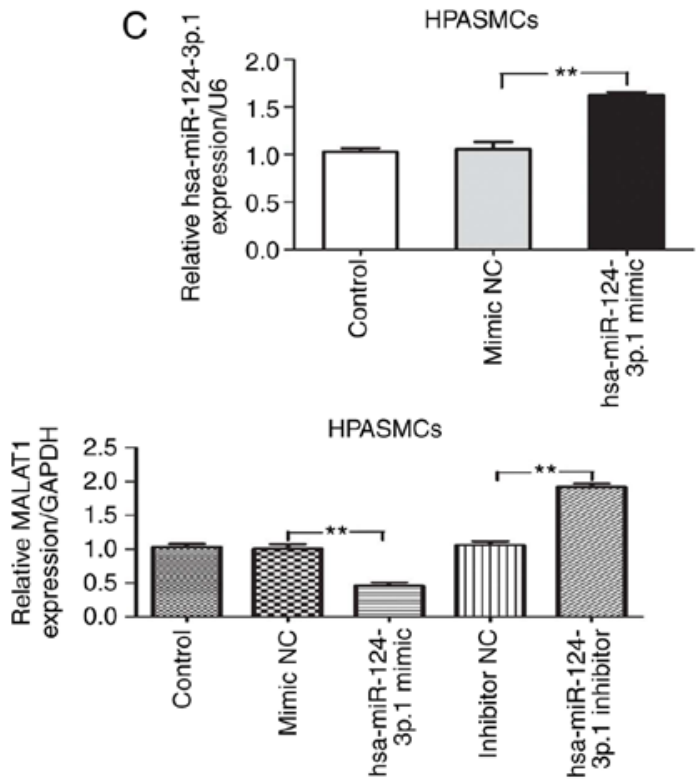

$\mathrm{F}$

WT MALAT1 6368...CAUCGCCACCCCGUGCCUUU...6388

hsa-miR-124-3p.1 3'...CCGUAAGUGGCGCACGGAAU...5

WH

Mut MALAT1 6368...CAUCGCCACCCCGTGGGTTU...6388

G

HPASMCs
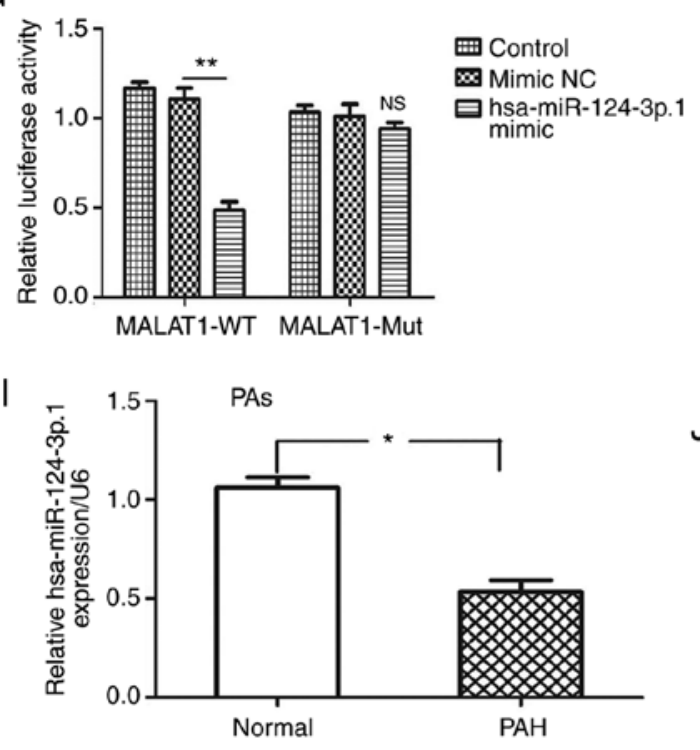

$\mathrm{H}$
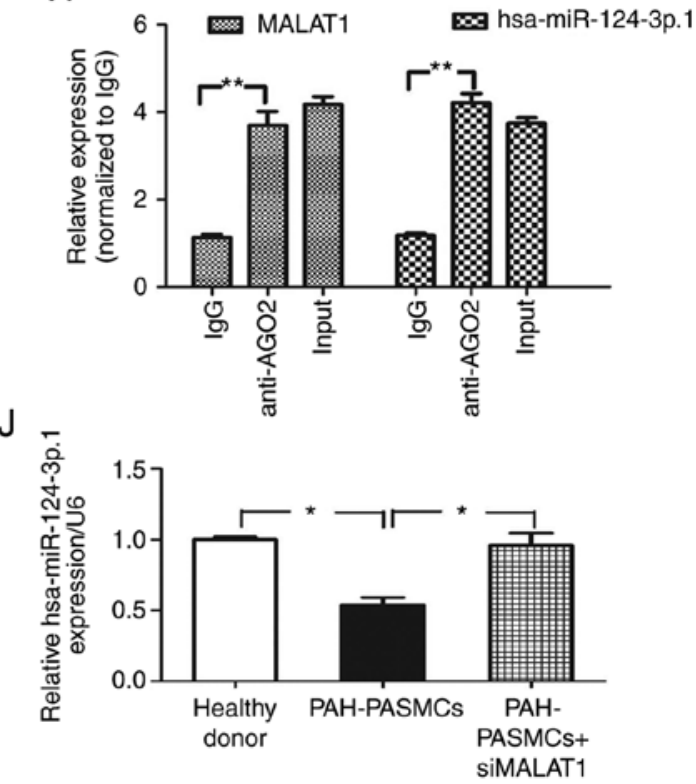

Figure 5. MALAT1 functions as a ceRNA for hsa-miR-124-3p.1. (A) Putative binding sites between MALAT1 and hsa-miR-124-3p.1. (B) hsa-miR-124-3p.1 expression in HPASMCs transfected with sh-MALAT1 or shNC was analyzed. Transfection efficiency of the (C) hsa-miR-124-3p.1 mimic or (D) inhibitor were determined by PCR. (E) Expression of MALAT1 in HPASMCs transfected with hsa-miR-124-3p.1 mimic or hsa-miR-124-3p.1 inhibitor. (F) Schematic showing the sequence alignment between hsa-miR-124-3p.1 and human MALAT1. Blue letters indicate the mutated seed region. (G) Luciferase reporter assay using HPASMCs to confirm whether MALAT1 directly binds with hsa-miR-124-3p.1. (H) RNA immunoprecipitation assay to investigate the interaction between MALAT1 and hsa-miR-124-3p.1. hsa-miR-124-3p.1 expression in (I) PA tissues and (J) HPASMCs, as detected by reverse transcription-quantitative PCR. ${ }^{*} \mathrm{P}<0.05$ and ${ }^{* *} \mathrm{P}<0.01$. NS, not significant; MALAT1, metastasis-associated lung adenocarcinoma transcript 1 ; ceRNA, competing endogenous RNA; miR, microRNA; HPASMCs, human pulmonary artery smooth muscle cells; PA, pulmonary artery; sh, short-hairpin RNA; NC, negative control.

features, such as abnormal cell proliferation and apoptosis resistance (24). Therefore, the authors of the present study hypothesized that MALAT1 may exert similar effects in PAH. The presence of a putative binding site for hsa-miR-124-3p.1 

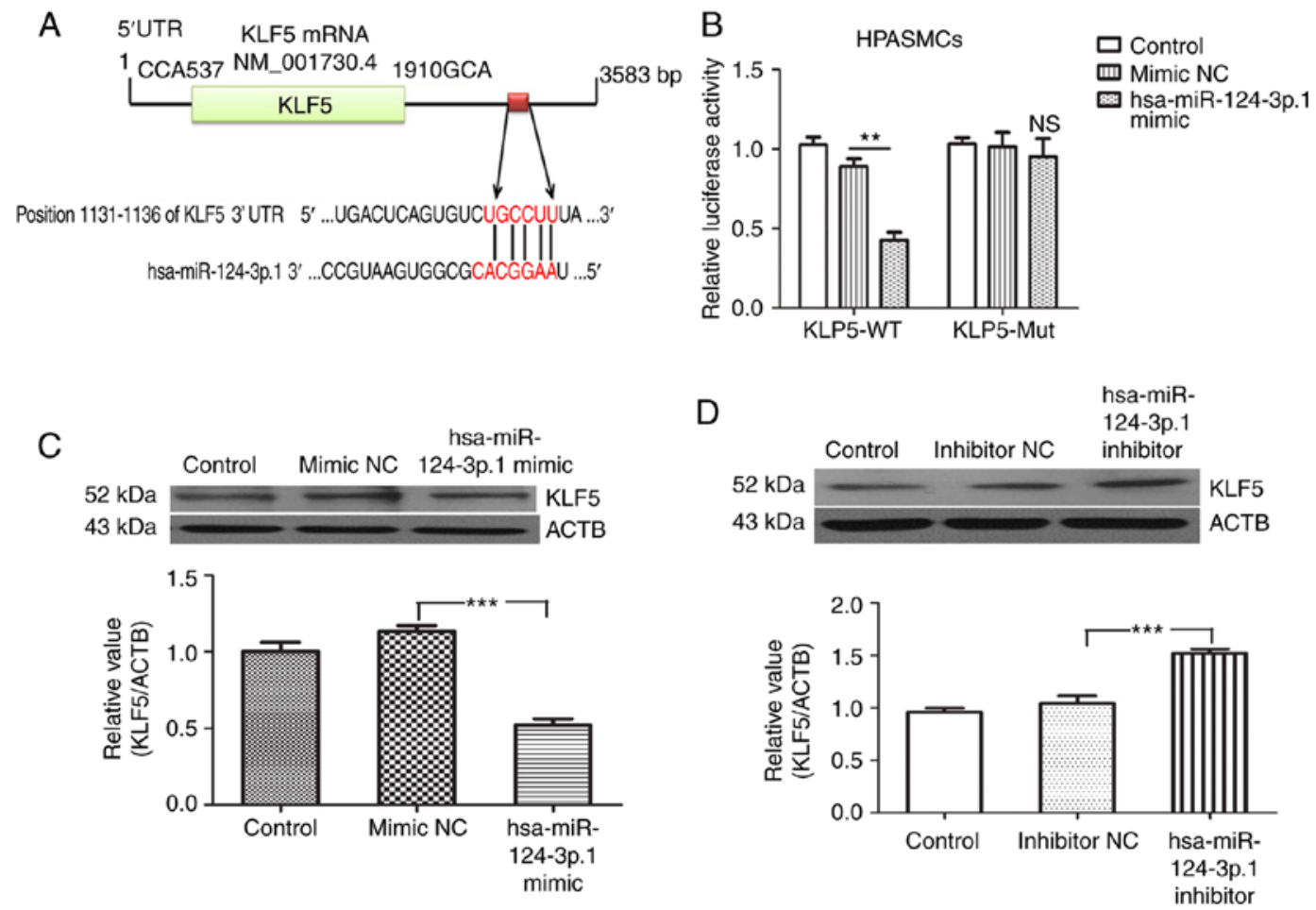

Figure 6. KLF5 is a direct target of hsa-miR-124-3p.1. (A) Sequence of the hsa-miR-124-3p.1 binding site in the 3'-UTR of KLF5. (B) Luciferase reporter assay using HPASMCs to examine whether KLF5 is a direct target of hsa-miR-124-3p.1. Protein expression levels of KLF5 in HPASMCs transfected with (C) hsa-miR-124-3p.1 mimic and (D) hsa-miR-124-3p.1 inhibitor. ${ }^{* *} \mathrm{P}<0.01$ and ${ }^{* * *} \mathrm{P}<0.001$; NS, not significant; KLF5, Kruppel-like factor 5; miR, microRNA; UTR, untranslated region; HPASMCs, human pulmonary artery smooth muscle cells; ACTB, $\beta$-actin; NC, negative control.

in the MALAT1 transcript was identified using LncBase Predicted v.2 (Fig. 5A). RT-qPCR experiments revealed that the expression of hsa-miR-124-3p.1 was significantly upregulated when MALAT1 was inhibited ( $\mathrm{P}<0.01$; Fig. 5B). The hsa-miR-124-3p.1 mimic or inhibitor was transfected into HPASMCs to examine the transfection efficiency (Fig. 5C and D). It was found that endogenous MALAT1 expression was significantly decreased in hsa-miR-124-3p.1 mimic-transfected cells $(\mathrm{P}<0.01)$, while transfection with the hsa-miR-124-3p.1 inhibitor increased MALAT1 expression $(\mathrm{P}<0.01$; Fig. 5E). These results indicate a negative correlation between MALAT1 and hsa-miR-124-3p.1. Luciferase reporter plasmids harboring wild-type MALAT1 and mutant MALAT1 3'-UTR sequences containing the predicted hsa-miR-124-3p.1 binding sites were generated (Fig. 5F). Dual luciferase reporter assays demonstrated that co-transfection of HPASMCs with the hsa-miR-124-3p.1 mimic resulted in a significant decrease in luciferase activity in cells transfected with wild-type MALAT1 $(\mathrm{P}<0.01)$, whereas the luciferase activity of cells transfected with the mutant form remained unaffected (Fig. 5G). The RIP assay results demonstrated that MALAT1 and hsa-miR-124-3p.1 were significantly enriched in AGO2 immunoprecipitates when compared with the IgG-pellet $(\mathrm{P}<0.01)$, indicating that MALAT1 and hsa-miR-124-3p.1 were located in the same RISC (Fig. 5H). This suggests that MALAT1 interacts with hsa-miR-124-3p.1 and functions as a ceRNA. Together, these findings prompted an investigation of the role of hsa-miR-124-3p.1 in PAH. Therefore, the expression of hsa-miR-124-3p.1 in 8 paired PAH and normal tissues were analyzed by RT-qPCR. The expression of hsa-miR-124-3p.1 was significantly reduced in PAH tissues when compared with normal PA tissues $(\mathrm{P}<0.01$; Fig. 5I). Subsequently, hsa-miR-124-3p.1 expression in HPASMCs derived from patients with PAH and healthy donors were compared. The results demonstrated that hsa-miR-124-3p.1 expression was significantly reduced in HPASMCs from patients with PAH when compared with healthy donors $(\mathrm{P}<0.05)$. This effect was significantly reversed when HPASMCs were transfected with shMALAT1 ( $\mathrm{P}<0.05$; Fig. 5J).

KLF5 is a direct target of hsa-miR-124-3p.1 and is aberrantly expressed in $P A$ tissues from patients with $P A H$ and hypoxic HPASMCs. To investigate the mechanism of action of hsa-miR-124-3p.1 in HPASMCs, bioinformatics analysis (http://www.targetscan.org/) was used to identify a complementary binding site between hsa-miR-124-3p.1 and the 3'-UTR of KLF5 (Fig. 6A). This suggested that KLF5 may be a target of hsa-miR-124-3p.1. Luciferase reporter plasmids containing the wild-type and mutant hsa-miR-124-3p.1 binding site sequences within the KLF5 3'-UTR were generated. Dual luciferase reporter assays demonstrated that co-transfection of HPASMCs with the hsa-miR-124-3p.1 mimic and wild-type sequences was associated with a significant decrease in luciferase activity $(\mathrm{P}<0.01)$, whereas no obvious changes in luciferase activity was observed in cells co-transfected with the mutant sequence (Fig. 6B). Western blotting analysis indicated that the expression of KLF5 was significantly reduced in hsa-miR-124-3p.1 mimic-transfected cells $(\mathrm{P}<0.01$; Fig. 6C). By contrast, KLF5 was observed to be significantly upregulated in the hsa-miR-124-3p.1 inhibitor-transfected group ( $\mathrm{P}<0.01$; Fig. 6D).

KLF5 has been reported to serve a crucial role in hypoxia-induced vascular remodeling (14). Therefore, to 
A

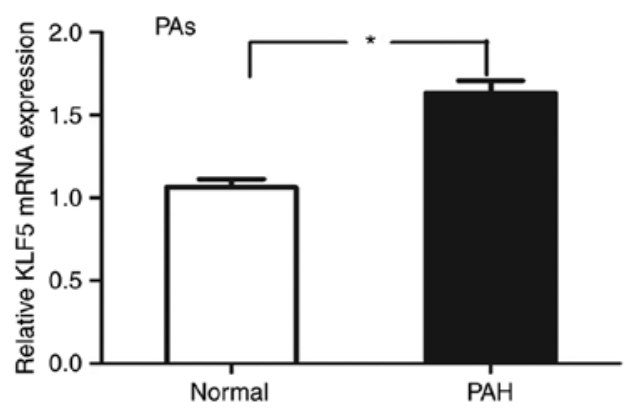

C
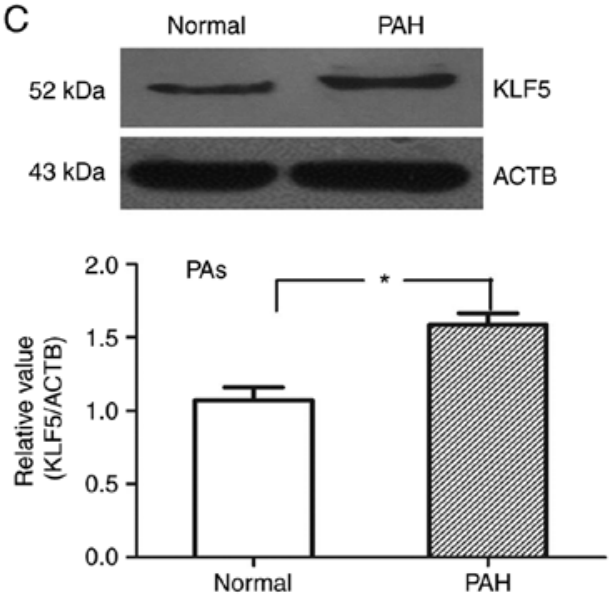

B
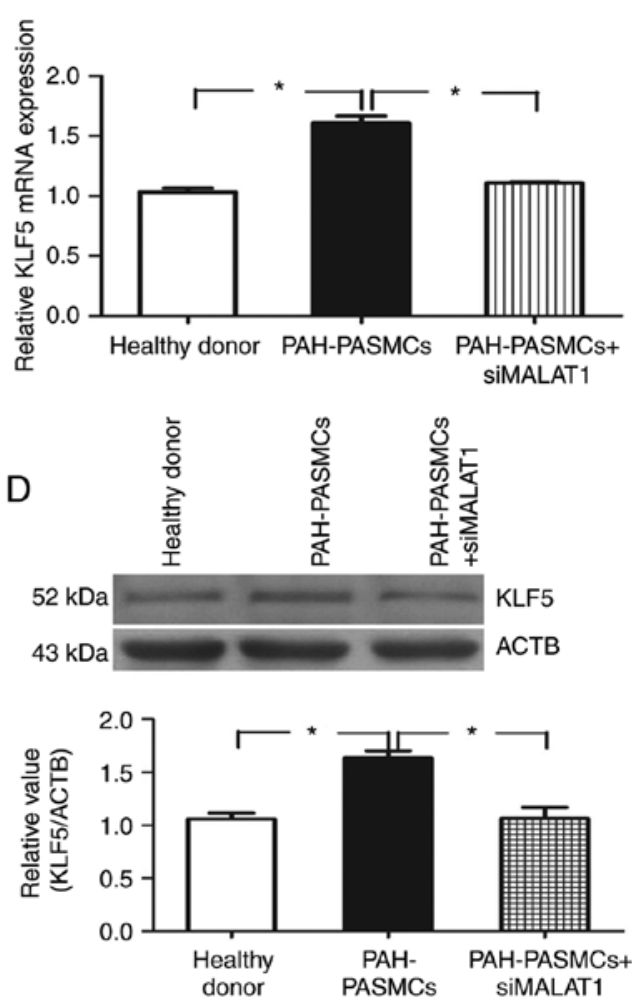

Figure 7. KLF5 is upregulated in the PA tissues and HPASMCs derived from patients with PAH. KLF5 mRNA expression in (A) PA tissues and (B) HPASMCs from healthy volunteers and PAH patients. KLF5 protein expression in (C) PA tissues and (D) HPASMCs from healthy volunteers and PAH patients. ${ }^{*}<0.05$. KLF5, Kruppel-like factor 5; PAH, pulmonary artery hypertension; ACTB, $\beta$-actin; PA, pulmonary artery; HPASMCs, human pulmonary artery smooth muscle cells.

further examine the role of KLF5 in PAH, the expression of KLF5 in the PAs derived from patients with PAH and healthy donors was examined. Western blotting and RT-qPCR results demonstrated significantly increased levels of KLF5 expression in the PAs from patients with PAH compared with healthy donors $(\mathrm{P}<0.05$; Fig. $7 \mathrm{~A}$ and $\mathrm{C})$. In parallel PA tissue experiments, KLF5 mRNA and protein expression levels were also significantly increased in HPASMCs derived from patients with PAH, and this effect was inhibited by transfection with shMALAT1 (P<0.01; Fig. 7B and D).

MALAT1 regulatesthe growthofHPASMCsviahsa-miR-124-3p.1 and KLF5. To examine the interaction between MALAT1, hsa-miR-124-3p.1 and KLF5, the expression of KLF5 protein in HPASMCs transfected with sh-MALAT1, hsa-miR-124-3p.1 mimic or inhibitors were analyzed. MALAT1 silencing reduced the expression of KLF5 at the mRNA and protein levels (Fig. 8A and B). The same effect on KLF5 expression was observed in cells transfected with hsa-miR-124-3p.1 mimics, whereas the hsa-miR-124-3p.1 inhibitor group showed the opposite effect. Of particular note, transfection with the hsa-miR-124-3p.1 inhibitor counteracted the downregulation of KLF5 expression induced by MALAT1 knockdown (Fig. 8C).

The effect of MALAT1 on the proliferation and migration of HPASMCs was largely offset by the miR-124-3p.1 mimic in MALAT1-silenced cells, while there was no obvious change in these characteristics in the control group and the MALAT1 plus miR-124-3p.1-silenced group. This suggests that MALAT1 knockdown was unable to suppress HPASMC proliferation and migration when hsa-miR-124-3p.1 was inhibited (Fig. 8D-I). The results indicate that MALAT1 silencing may inhibit the growth and migration of HPASMCs by sponging hsa-miR-124-3p.1. Ultimately, these results demonstrate that MALAT1 may regulate KLF5 expression by binding competitively to hsa-miR-124-3p, thus promoting the growth of HASMCs.

\section{Discussion}

Pulmonary vascular remodeling is a multifactorial pathological process characterized by the abnormal proliferation and migration of HPASMCs, as well as the resistance of these cells to apoptosis. The identification of suitable therapeutic targets that suppress vascular remodeling is important. Emerging evidence has increased understanding of the multifaceted role of lncRNAs as modifying factors of vascular remodeling. For instance, MALAT1 (7) and transforming growth factor- $\beta 2$-overlapping transcript 1 (25) have been found to promote vascular endothelial cell dysfunction, while long intergenic RNA-p21 (26), lnc-Ang362 (27) and maternally expressed 3 (19) contribute to vascular smooth muscle cell proliferation. MALAT1 was originally discovered in non-small cell lung cancer and is used as a prognostic marker for lung cancer metastasis $(4,28)$. Previous studies have reported that MALAT1 is aberrantly expressed in numerous 


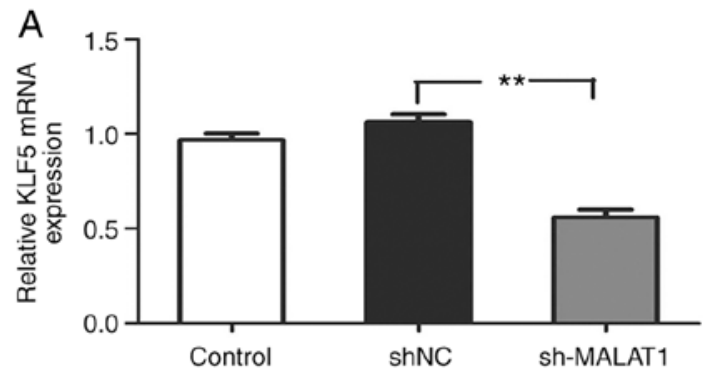

B
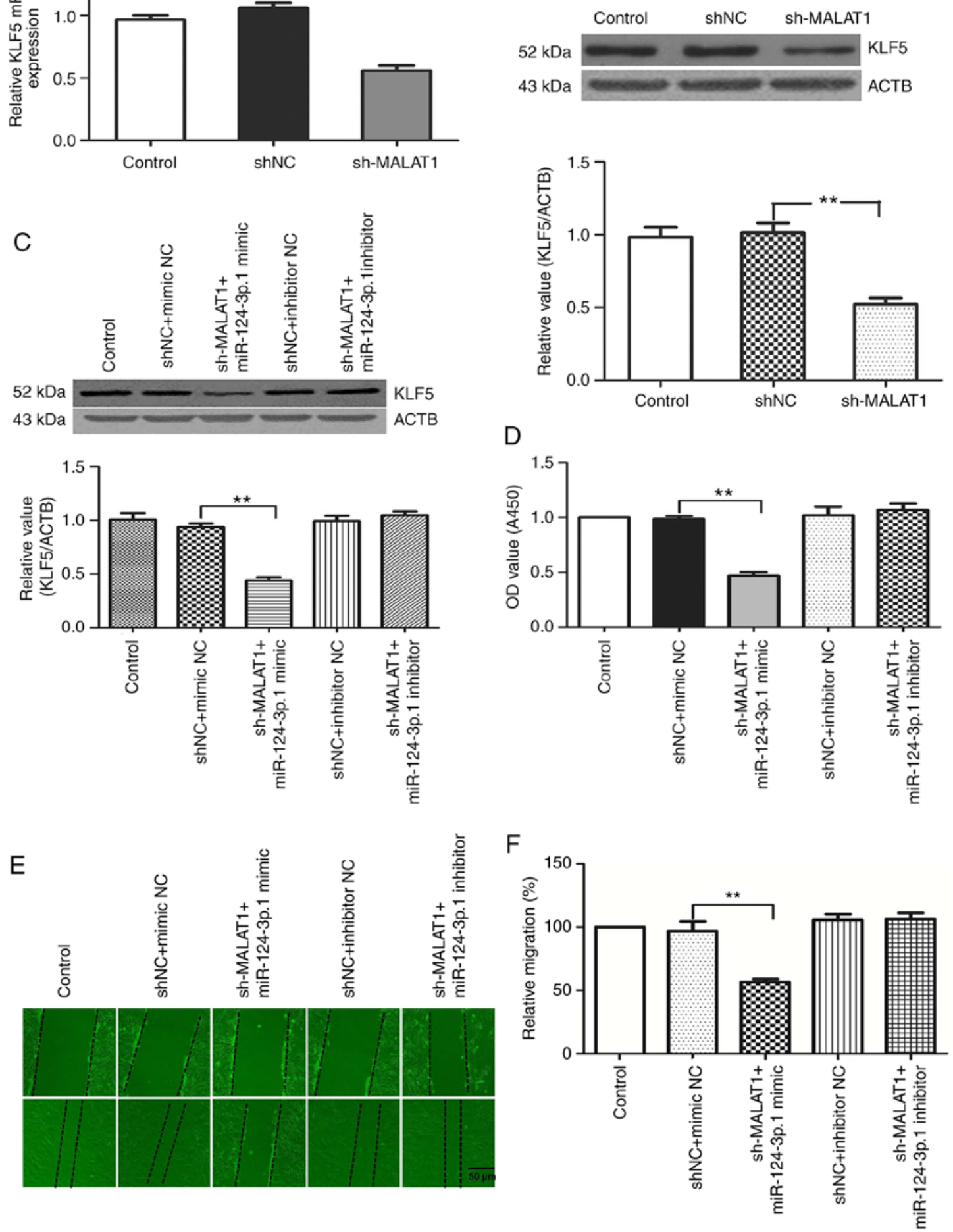

Figure 8. MALAT1 knockdown suppresses the expression of KLF5 via upregulating hsa-miR-124-3p.1. KLF5 (A) mRNA and (B) protein expression levels in HPASMCs transfected with sh-MALAT1. (C) Protein expression levels of KLF5 in HPASMCs co-transfected with sh-MALAT1 and hsa-miR-124-3p.1 mimic or inhibitor. HPASMCs were co-transfected with (D) sh-MALAT1 mimic or inhibitor. HPASMCs were co-transfected with (E) hsa-miR-124-3p.1 mimic or inhibitor. Changes in (D) cell viability and (E) migration were assessed and (F) analyzed.

solid tumors and is involved in the proliferation and metastasis of several cancers $(29,30)$. However, accumulating evidence suggests that MALAT1 may also regulate multiple pathological vascular remodeling processes $(7,8)$. However, the role and precise mechanisms of MALAT1 in pulmonary vascular remodeling is not fully understood. The present study reports that MALAT1 is upregulated in the PA tissues and HPASMCs derived from patients with PAH. In addition, knockdown of MALAT1 reduced HPASMC viability and proliferation, with a greater number of cells in the $G_{0} / G_{1}$ phase. Bioinformatics analysis predicted an interaction between MALAT1 and hsa-miR-124-3p.1. A negative regulatory feedback loop 

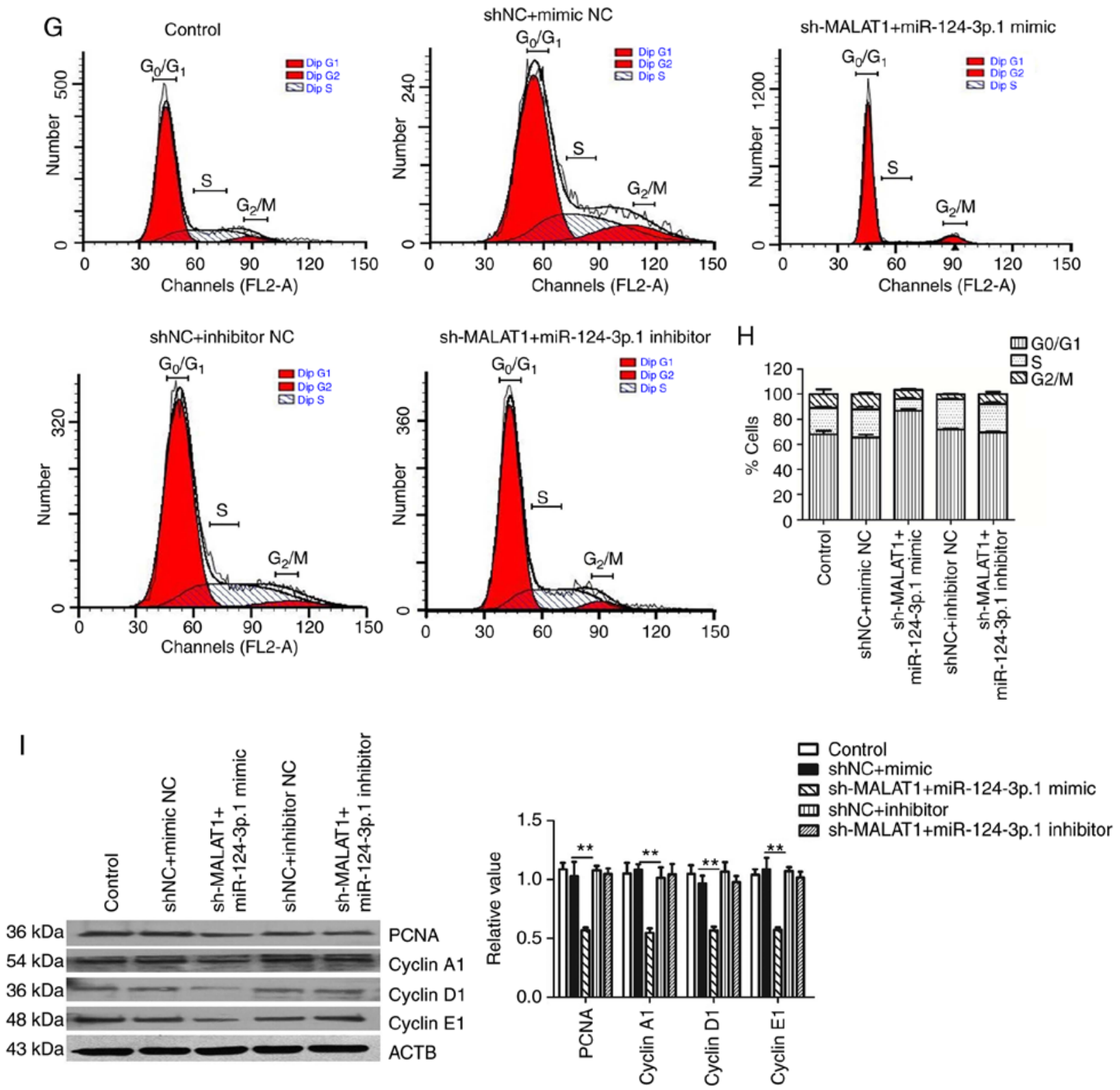

Figure 8. Continued. (G) Cell cycle analysis of HPASMCs co-transfected with sh-MALAT1 and hsa-miR-124-3p.1 mimic or inhibitor. (H) Histogram presenting the percentage of cells in the $\mathrm{G}_{0} / \mathrm{G}_{1}, \mathrm{~S}$ and $\mathrm{G}_{2} / \mathrm{M}$ phases. (I) Protein expression levels of PCNA, cyclin A1, cyclin D1 and cyclin E1 were analyzed by western blotting following co-transfection of HPASMCs with sh-MALAT1 and hsa-miR-124-3p.1 mimic or inhibitor. ${ }^{* *} \mathrm{P}<0.01$. MALAT1, metastasis-associated lung adenocarcinoma transcript 1; KLF5, Kruppel-like factor 5; miR, microRNA; HPASMCs, human pulmonary artery smooth muscle cells; shRNA, short-hairpin RNA; PCNA, proliferating cell nuclear antigen; ACTB, $\beta$-actin; NC, negative control.

between MALAT1 and hsa-miR-124-3p.1 was also subsequently identified, and the function of MALAT1 as a ceRNA by sponging hsa-miR-124-3p.1 in HPASMCs was demonstrated.

A number of previous studies have reported that increased expression of MALAT1 may serve an important role in the development of different tumors $(4,5,31)$. In addition, MALAT1 was reported to be an important regulator of angiogenesis (7). The present study primarily focused on the effects of MALAT1 on pulmonary vascular remodeling and found that MALAT1 expression was increased in PA tissues and HPASMCs derived from patients with PAH. These results are consistent with the results of a previous study (8). In the present study, knockdown of MALAT1 in HPASMCs suppressed viability and inhibited cell cycle progression through the $\mathrm{G}_{0} / \mathrm{G}_{1}$ phase. The protein expression levels of PCNA, cyclin A1, cyclin D1 and cyclin E1 were also downregulated, which is consistent with the cell cycle analysis results. By contrast, these effects were reversed following overexpression of MALAT1.

Previous studies have demonstrated that numerous lncRNAs function as ceRNAs, which inhibit normal miRNA activity and modulate miRNA signaling pathways (32-34). Therefore, the authors of the current study proposed that an association between MALAT1 and miRNA in PAH may exist. Bioinformatics analysis revealed multiple miRNA binding sites in the MALAT1 sequence. However, RT-qPCR results indicated that only hsa-miR-124-3p.1 was able to repress the expression of MALAT1 RNA in HPASMCs. These results were supported by luciferase reporter and RIP assay analyses. Therefore, the present study provides strong evidence to suggest that MALAT1 may function as a ceRNA for hsa-miR-124-3p.1. 
In addition, a negative correlation between MALAT1 and hsa-miR-124-3p.1 in PAH progression was observed.

Previous studies have demonstrated that miR-124 affects the inflammatory phenotype, proliferation and migration of pulmonary vascular fibroblasts $(35,36)$. Consistently, the results of the current study demonstrated that hsa-miR-124-3p.1 was downregulated in PA tissues and HPASMCs derived from patients with PAH, implying that the expression of hsa-miR-124-3p.1 may be involved in the development PAH. KLF5 was also identified as a direct functional effector of hsa-miR-124-3p.1. A series of reports have demonstrated that KLF5 is elevated in human lung biopsies and HPASMCs isolated from the distal PAs of patients with PAH when compared with those obtained from normal patients $(14,37,38)$. The results of the current study provide evidence to suggest that MALAT1 may function as a ceRNA for hsa-miR-124-3p.1. In addition, the expression levels of MALAT1 were observed to be downregulated in HPASMCs transfected with hsa-miR-124-3p.1 mimics, while its expression was upregulated in the hsa-miR-124-3p.1 inhibitor-transfected group of cells. Furthermore, luciferase assays demonstrated that hsa-miR-124-3p.1 reduced the activity of the wild-type MALAT1 3'-UTR but had no effect on the mutant sequence. The results of the RIP assay indicated that MALAT1 and hsa-miR-124-3p.1 formed part of the RISC. Together, these results suggest that MALAT1 functions as a ceRNA and interacts with hsa-miR-124-3p.1. As such, MALAT may promote pulmonary vascular remodeling progression in vivo by operating as a ceRNA.

It was found that MALAT1, a ceRNA, shared the complementary sequence of hsa-miR-124-3p.1 with its target KLF5 by bioinformatics software analysis. In addition, the expression of KLF5 was observed to be influenced by MALAT1 in HPASMCs. MALAT1 silencing reduced the mRNA and protein levels of KLF5. The effect of MALAT1 on KLF5 expression was reversed by transfection of cells with the hsa-miR-124-3p.1 inhibitor. In addition, KLF5 expression was downregulated when HPASMCs were co-transfected with sh-MALAT1 and hsa-miR-124-3p.1 mimics, while hsa-miR-124-3p.1 suppression exhibited the opposite effects. Furthermore, silencing of MALAT1 expression and overexpression of hsa-miR-124-3p.1 inhibited the proliferation and migration of HPASMCs. Taken together, the results suggest that the MALAT1/hsa-miR-124-3p.1/KLF5 axis may serve an important role in the development of PAH.

In conclusion, the expression of MALAT1,hsa-miR-124-3p.1 and KLF5 in HPASMCs and PA tissues were examined in the present study. MALAT1 promoted the growth and migration of HPASMCs potentially via sponging hsa-miR-124-3p.1 and KLF5. Understanding the molecular mechanisms of MALAT1 in PAH may provide a novel approach for developing effective therapeutic interventions for patients with PAH.

\section{Acknowledgements}

Not applicable.

\section{Funding}

This study was supported by National Natural Science Foundation of China (grant no. 81500039).

\section{Availability of data and materials}

All data generated or analyzed during this study are included in this published article.

\section{Authors' contributions}

DW performed H\&E staining, the Cell Counting Kit-8 assay, luciferase reporter gene activity assay and RNA immunoprecipitation assay, and wrote the manuscript. HX conducted western blotting and reverse transcription-quantitative polymerase chain reaction experiments. BW performed flow cytometry assays. SJ performed cell culture, cell transfection and the wound healing assay. HP was responsible for patient sample collection and acquisition of clinical data. RW designed the present study and the experiments. JC interpreted and analyzed the data. All authors read and approved the final version of the manuscript.

\section{Ethics approval and consent to participate}

The present study was approved by the Ethics Committee for the use of human samples of Wuxi People's Hospital Affiliated to Nanjing Medical University (Wuxi, China), which was in accordance with the code of ethics of the Declaration of Helsinki developed by the World Medical Association. Each individual provided written informed consent prior to their participation.

\section{Patient consent for publication}

Not applicable.

\section{Competing interests}

The authors declare that they have no conflict of interest.

\section{References}

1. Voelkel NF, Gomez-Arroyo J, Abbate A, Bogaard HJ and Nicolls MR: Pathobiology of pulmonary arterial hypertension and right ventricular failure. The Euro Respir J 40: 1555-1565, 2012.

2. Uchida S and Dimmeler S: Long noncoding RNAs in cardiovascular diseases. Circ Res 116: 737-750, 2015.

3. Zhao XY and Lin JD: Long noncoding RNAs: A new regulatory code in metabolic control. Trends Biochem Sci 40: 586-596, 2015.

4. Ji P, Diederichs S, Wang W, Böing S, Metzger R, Schneider PM, Tidow N, Brandt B, Buerger H, Bulk E, et al: MALAT-1, a novel noncoding RNA, and thymosin beta4 predict metastasis and survival in early-stage non-small cell lung cancer. Oncogene 22: 8031-8041, 2003

5. Ren S, Liu Y, Xu W, Sun Y, Lu J, Wang F, Wei M, Shen J, Hou J, Gao X, et al: Long noncoding RNA MALAT-1 is a new potential therapeutic target for castration resistant prostate cancer. J Urol 190: 2278-2287, 2013.

6. Qi Y, Ooi HS, Wu J, Chen J, Zhang X, Tan S, Yu Q, Li YY, Kang Y, Li H, et al: MALAT1 long ncRNA promotes gastric cancer metastasis by suppressing PCDH10. Oncotarget 7: 12693-12703, 2016.

7. Michalik KM, You X, Manavski Y, Doddaballapur A, Zörnig M, Braun T, John D, Ponomareva Y, Chen W, Uchida S, et al: Long noncoding RNA MALAT1 regulates endothelial cell function and vessel growth. Circ Res 114: 1389-1397, 2014.

8. Brock M, Schuoler C, Leuenberger C, Bühlmann C, Haider TJ, Vogel J, Ulrich S, Gassmann M, Kohler M and Huber LC: Analysis of hypoxia-induced noncoding RNAs reveals metastasis-associated lung adenocarcinoma transcript 1 as an important regulator of vascular smooth muscle cell proliferation. Exp Biol Med (Maywood) 242: 487-496, 2017. 
9. Lund E, Guttinger S, Calado A, Dahlberg JE and Kutay U: Nuclear export of microRNA precursors. Science 303: 95-98, 2004.

10. Wang X, Li M, Wang Z, Han S, Tang X, Ge Y, Zhou L, Zhou C, Yuan $Q$ and Yang M: Silencing of long noncoding RNA MALAT1 by miR-101 and miR-217 inhibits proliferation, migration, and invasion of esophageal squamous cell carcinoma cells. J Biol Chem 290: 3925-3935, 2015.

11. Han Y, Liu Y, Zhang H, Wang T, Diao R, Jiang Z, Gui Y and Cai Z: Hsa-miR-125b suppresses bladder cancer development by down-regulating oncogene SIRT7 and oncogenic long non-coding RNA MALAT1. FEBS Lett 587: 3875-3882, 2013.

12. Yu F, Lu Z, Cai J, Huang K, Chen B, Li G, Dong P and Zheng J: MALAT1 functions as a competing endogenous RNA to mediate Rac1 expression by sequestering miR-101b in liver fibrosis. Cell Cycle 14: 3885-3896, 2015

13. Sun Y, Cheng H, Wang G, Yu G, Zhang D, Wang Y, Fan W and Yang W: Deregulation of miR-183 promotes melanoma development via lncRNA MALAT1 regulation and ITGB1 signal activation. Oncotarget 8: 3509-3518, 2017.

14. Li X,He Y, Xu Y, Huang X,Liu J,Xie M and Liu X: KLF5 mediates vascular remodeling via HIF-1 $\alpha$ in hypoxic pulmonary hypertension. Am J Physiol Lung Cell Mol Physiol 310: L299-L310, 2016.

15. Nie X, Dai Y, Tan J, Chen Y, Qin G, Mao W, Zou J, Chang Y, Wang $Q$ and Chen J: $\alpha$-Solanine reverses pulmonary vascular remodeling and vascular angiogenesis in experimental pulmonary artery hypertension. J Hypertens 35: 2419-2435, 2017.

16. Mao W, Xia W and Chen J: Interobserver variability in grading acute rejection after lung transplantation. Chest 145: 416-417, 2014

17. Nie X, Qin G, Mao W, Wang W, Chang Y, Wei D, Zhou M, Wu B and Chen J: Axis inhibition protein 2 deficiency leads to hypoxic pulmonary hypertension through beta-catenin signaling pathway. J Hypertens 34: 877-892, 2016.

18. Peng G, Xu J, Liu R, Fu Z, Li S, Hong W, Chen J, Li B and Ran P: Isolation, culture and identification of pulmonary arterial smooth muscle cells from rat distal pulmonary arteries. Cytotechnology 69: 831-840, 2017.

19. Sun Z, Nie X, Sun S, Dong S, Yuan C, Li Y, Xiao B, Jie D and Liu Y: Long non-coding RNA MEG3 downregulation triggers human pulmonary artery smooth muscle cell proliferation and migration via the p53 signaling pathway. Cell Physio Biochem 42: 2569-2581, 2017.

20. Livak KJ and Schmittgen TD: Analysis of relative gene expression data using real-time quantitative PCR and the 2(-Delta Delta C(T)) method. Methods 25: 402-408, 2001.

21. Liu Y, Ma C, Zhang Q, Yu L, Ma J, Zhang L, Hao X, Cao F Wang $\mathrm{L}$ and $\mathrm{Zhu} \mathrm{D}$ : The key role of transforming growth factor-beta receptor I and 15-lipoxygenase in hypoxia-induced proliferation of pulmonary artery smooth muscle cells. Int J Biochem Cell Biol 44: 1184-1202, 2012.

22. Guppy A, Jamal-Hanjani M and Pickering L: Anticancer effects of metformin and its potential use as a therapeutic agent for breast cancer. Future Oncol 7: 727-736, 2011

23. Qu J, Li M, Zhong W and Hu C: Competing endogenous RNA in cancer: A new pattern of gene expression regulation. Int J Clin Exp Med 8: 17110-17116, 2015.

24. Voelkel NF, Cool C, Lee SD, Wright L, Geraci MW and Tuder RM: Primary pulmonary hypertension between inflammation and cancer. Chest 114 (Suppl 3): 225S-230S, 1998.

25. Huang S, Lu W, Ge D, Meng N, Li Y, Su L, Zhang S, Zhang Y, Zhao B and Miao J: A new microRNA signal pathway regulated by long noncoding RNA TGFB2-OT1 in autophagy and inflammation of vascular endothelial cells. Autophagy 11: 2172-2183, 2015.
26. Wu G, Cai J, Han Y, Chen J, Huang ZP, Chen C, Cai Y, Huang H, Yang Y, Liu Y, et al: LincRNA-p21 regulates neointima formation, vascular smooth muscle cell proliferation, apoptosis, and atherosclerosis by enhancing p53 activity. Circulation 130: 1452-1465, 2014.

27. Leung A, Trac C, Jin W, Lanting L, Akbany A, Sætrom P Schones DE and Natarajan R: Novel long noncoding RNAs are regulated by angiotensin II in vascular smooth muscle cells. Circ Res 113: 266-278, 2013

28. Sun Y and Ma L: New insights into long non-coding RNA MALAT1 in cancer and metastasis. Cancers (Basel) 11: E216, 2019.

29. Wang SH, Zhang WJ, Wu XC, Weng MZ, Zhang MD, Cai Q, Zhou D, Wang JD and Quan ZW: The IncRNA MALAT1 functions as a competing endogenous RNA to regulate MCL-1 expression by sponging miR-363-3p in gallbladder cancer. J Cell Mol Med 20: 2299-2308, 2016.

30. Li L, Chen H, Gao Y, Wang YW, Zhang GQ, Pan SH, Ji L, Kong R, Wang G, Jia YH, et al: Long noncoding RNA MALAT1 promotes aggressive pancreatic cancer proliferation and metastasis via the stimulation of autophagy. Mol Cancer Ther 15: 2232-2243, 2016

31. Wu XS, Wang XA, Wu WG, Hu YP, Li ML, Ding Q, Weng H, Shu YJ, Liu TY and Jiang L, et al: MALAT1 promotes the proliferation and metastasis of gallbladder cancer cells by activating the ERK/MAPK pathway. Cancer Biol Ther 15: 806-814, 2014.

32. Poliseno L, Salmena L, Zhang J, Carver B, Haveman WJ and Pandolfi PP: A coding-independent function of gene and pseudogene mRNAs regulates tumour biology. Nature 465: 1033-1038, 2010.

33. Cesana M, Cacchiarelli D, Legnini I, Santini T, Sthandier O, Chinappi M, Tramontano A and Bozzoni I: A long noncoding RNA controls muscle differentiation by functioning as a competing endogenous RNA. Cell 147: 358-369, 2011.

34. Ebert MS and Sharp PA: MicroRNA sponges: Progress and possibilities. RNA 16: 2043-2050, 2010.

35. Wang D, Zhang H, Li M, Frid MG, Flockton AR, McKeon BA, Yeager ME, Fini MA, Morrell NW, Pullamsetti SS, et al: MicroRNA-124 controls the proliferative, migratory, and inflammatory phenotype of pulmonary vascular fibroblasts. Circ Res 114: 67-78, 2014.

36. Kang K, Peng X, Zhang X, Wang Y, Zhang L, Gao L, Weng T, Zhang H, Ramchandran R, Raj JU, et al: MicroRNA-124 suppresses the transactivation of nuclear factor of activated $\mathrm{T}$ cells by targeting multiple genes and inhibits the proliferation of pulmonary artery smooth muscle cells. J Biol Chem 288: 25414-25427, 2013.

37. Courboulin A, Tremblay VL, Barrier M, Meloche J, Jacob MH, Chapolard M, Bisserier M, Paulin R, Lambert C, Provencher S and Bonnet S: Krüppel-like factor 5 contributes to pulmonary artery smooth muscle proliferation and resistance to apoptosis in human pulmonary arterial hypertension. Respir Res 12: 128, 2011.

38. Abe K, Sugiura H, Hashimoto Y, Ichikawa T, Koarai A Yamada M, Numakura T, Onodera K, Tanaka R, Sato K, et al: Possible role of Krüppel-like factor 5 in the remodeling of small airways and pulmonary vessels in chronic obstructive pulmonary disease. Respir Res 17: 7, 2016.

This work is licensed under a Creative Commons Attribution-NonCommercial-NoDerivatives 4.0 International (CC BY-NC-ND 4.0) License. 